\title{
Superficial Siderosis Misdiagnosed As Parkinson's Disease in a 70-year-old Male Breast Cancer Survivor
}

\author{
Stephen J. Bordes ${ }^{1}$, Katrina E. Bang ${ }^{2}$, R. Shane Tubbs ${ }^{3,2}$ \\ 1. Department of Anatomical Sciences, St. George's University School of Medicine, St. George's, GRD 2. Department of \\ Anatomical Sciences, St. George's University, St. George's, GRD 3. Departments of Neurosurgery and Structural \& \\ Cellular Biology, Tulane University \& Ochsner Clinic Neurosurgery Program, Tulane University School of Medicine, \\ New Orleans, USA
}

Corresponding author: Stephen J. Bordes, sbordes@sgu.edu

\begin{abstract}
A 70-year-old African American male with a history of hypertension, congestive heart failure, breast cancer status-post six rounds of doxorubicin/cyclophosphamide, and Parkinson's disease managed with carbidopa/levodopa presented to the emergency department with bilateral hearing loss and ataxia. The patient was admitted and evaluated for possible traumatic, oncological, and pharmacological etiologies. Further investigation revealed hypointensities along the cerebellar folia and basal cisterns on MRI in addition to the two-year history of progressive bilateral hearing loss and gait ataxia. In view of these findings, the patient was diagnosed with superficial siderosis and Parkinson's medications were discontinued. Superficial siderosis should be considered as a diagnosis in cases of bilateral hearing loss and ataxia in patients with history of anticoagulation and risk factors for prior cerebrovascular accidents or head trauma.
\end{abstract}

Categories: Internal Medicine, Neurology

Keywords: superficial siderosis, parkinson's disease, ataxia, bilateral hearing loss

\section{Introduction}

Superficial siderosis is a rare neurological disease associated with chronic subpial deposition of hemosiderin throughout the brain and spinal cord due to recurrent episodes of subarachnoid hemorrhage [1-9]. Disease prevalence within the general population remains unclear, although population-based studies have reported a range of $0.21 \%-1.43 \%$ in patients aged over 55 years, with greater prevalence in those aged over 69 years $[2,10]$. The disease presents clinically as a triad of bilateral sensorineural hearing loss, ataxia, and myelopathy with pathognomonic findings of hypointensities along the brainstem, cerebellum, and spinal cord identified by multisequence MRI.

Received 03/03/2020

Review began 03/13/2020 Review ended 03/15/2020 Published 03/18/2020

๑) Copyright 2020 Bordes et al. This is an open access article distributed under the terms of the Creative Commons Attribution License CC-BY 4.0., which permits unrestricted use, distribution, and reproduction in any medium, provided the original author and source are credited.
In view of the nonspecific sensorineural, neurological, and cerebrovascular findings associated with superficial siderosis combined with its low prevalence within the population, the disease can often be misdiagnosed or missed altogether. Herein, we present a case of superficial siderosis that was misdiagnosed and mistreated as Parkinson's disease. We suggest that in order to avoid future misdiagnoses, superficial siderosis should be considered as a differential diagnosis for elderly patients, especially those on anticoagulation or with a history of brain trauma or injury, who present with bilateral sensorineural hearing loss and gait ataxia.

\section{Case Presentation}

A 70-year-old African American man was admitted to the hospital with bilateral hearing loss and ataxia. The patient was initially brought in by his wife owing to concern for a potential traumatic brain injury, as he had hit his head on a metal gate three days previously while working on his farm. Upon further inquiries concerning history, the patient's wife stated that his hearing and gait had progressively declined over the previous two years. The patient had first struggled with high pitched sounds, followed by both high- and low-pitched sounds. His wife noticed him sitting closer to his television, struggling to converse in loud settings, and asking others to repeat themselves more frequently. Both the patient and his wife attributed the initial hearing losses to old age.

The patient was diagnosed six years earlier at an outside facility with obstructive sleep apnea, hypertension, benign prostatic hyperplasia, and breast cancer. At that time, he underwent a bilateral mastectomy followed by six months of chemotherapy with doxorubicin and cyclophosphamide. He was subsequently diagnosed with deep vein thrombosis, pulmonary embolism, and congestive heart failure attributed to the chemotherapy. Long-term anticoagulation with warfarin was initiated. The remainder of his medications included lisinopril, metoprolol, amlodipine, tamsulosin, anastrozole, and tamoxifen. The patient followed up consistently with his primary physician and oncologist. At an appointment two years earlier, his gait was 


\section{Cureus}

noted to be in decline with a tendency to lose balance, and his movements were slowed. He was inappropriately diagnosed with Parkinson's disease, and carbidopa/levodopa therapy was initiated due to the similarity of physical manifestations between the patient's presentation and the misdiagnosed movement disorder.

Presently, exam findings on presentation were unremarkable with the exception of bilateral sensorineural hearing loss, ataxia, and 1+ pitting edema to the anterior tibia bilaterally. Weber testing showed no lateralization and Rinne testing revealed air conduction greater than bone conduction. The patient had no signs of trauma or infection to the external ear or tympanum bilaterally. There was no evidence of a bulging membrane. The light reflex was observed. Cranial nerves (CNs) II-XII were otherwise grossly intact. The patient's gait was markedly ataxic and spastic with a tendency to fall. Romberg testing was negative. He did not exhibit a resting tremor or cogwheeling of the extremities. Head CT without contrast was ordered, which showed no acute intracranial abnormalities; however, old bilateral basal ganglia infarcts were noted. Twelve-lead ECG showed normal sinus rhythm with a first-degree atrioventricular block (Figure 1).

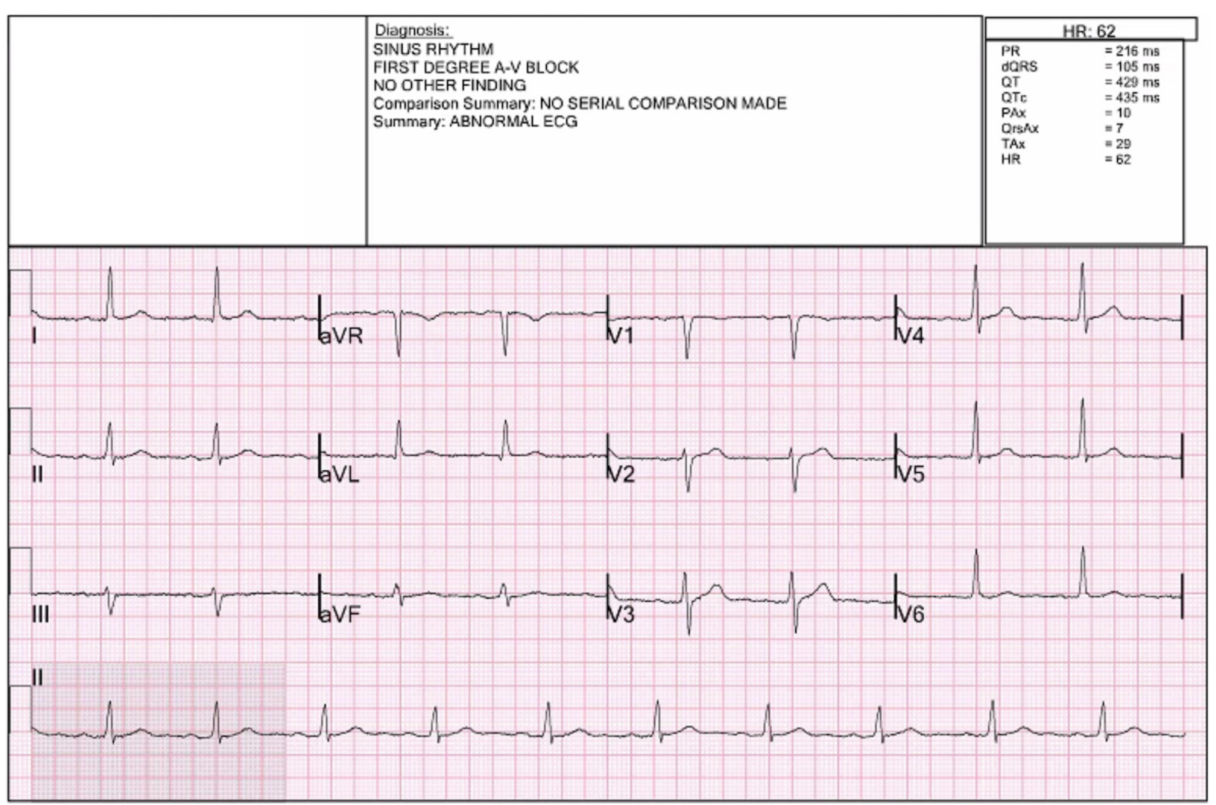

\section{FIGURE 1: Twelve-lead ECG}

HR: heart rate; A-V: atrioventricular; ECG: electrocardiogram; Ax; axis

Remaining abbreviations cannot be expanded and refer to ECG waves and intervals.

Multisequence brain MRI showed no signs of acute stroke; however, old blood products consistent with the hypointensity of hemosiderin were noted along the cerebellar folia and basal cisterns (Figure 2). 


\section{Cureus}
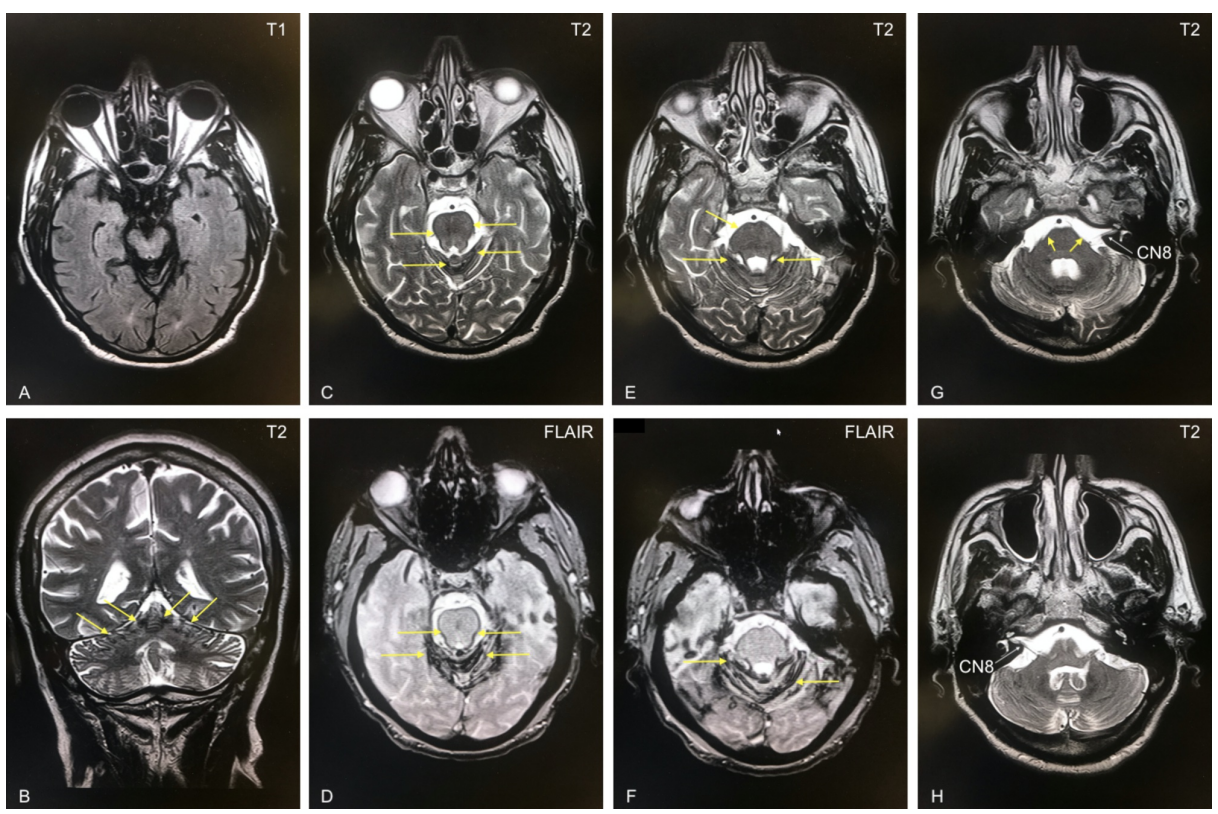

\section{FIGURE 2: Brain MRI}

Axial plane multisequence T1, T2, and FLAIR MRI showing the midbrain (A), pons (C-F), and cerebellum (G, H). Coronal plane T2-weighted MRI (B).

Hemosiderin hypointensities (black) noted along the cerebellar folia and basal cisterns in both coronal and axial planes (yellow arrows). Cranial nerve VIII (CN8) noted (white arrows).

FLAIR, fluid-attenuated inversion recovery

Periventricular small vessel disease and parenchymal atrophy were also noted. Carotid artery and renal ultrasounds showed no evidence of occlusion, obstruction, plaque deposition, or hydronephrosis. Transthoracic echocardiography showed an ejection fraction of $60 \%$, trivial mitral regurgitation, and physiological pulmonary regurgitation (Table 1). There was no sign of ventricular abnormality or dysfunction. 


\section{Cureus}

\begin{tabular}{|c|c|c|c|c|c|c|c|c|c|}
\hline \multicolumn{2}{|c|}{ EF Findings } & \multicolumn{4}{|c|}{ M-Mode/2D Measurements and Calculations } & \multicolumn{4}{|c|}{ Doppler Measurements and Calculations } \\
\hline $\begin{array}{l}\text { EF } \\
\text { calculated }\end{array}$ & $60.2 \%$ & $\begin{array}{l}\text { LV diastolic } \\
\text { dimension }\end{array}$ & $5.45 \mathrm{~cm}$ & $\begin{array}{l}\text { LV systolic } \\
\text { dimension }\end{array}$ & $\begin{array}{l}4.01 \\
\mathrm{~cm}\end{array}$ & $\begin{array}{l}\text { MV peak E- } \\
\text { wave }\end{array}$ & $0.51 \mathrm{~m} / \mathrm{s}$ & $\begin{array}{l}\mathrm{AV} \text { peak } \\
\text { velocity }\end{array}$ & $1.22 \mathrm{~m} / \mathrm{s}$ \\
\hline & & LV PW diastolic & $1.02 \mathrm{~cm}$ & $\begin{array}{l}\text { LV volume } \\
\text { systolic }\end{array}$ & $\begin{array}{l}64.5 \\
\mathrm{~mL}\end{array}$ & $\begin{array}{l}\text { MV peak A- } \\
\text { wave }\end{array}$ & $0.58 \mathrm{~m} / \mathrm{s}$ & $\begin{array}{l}\text { AV peak } \\
\text { gradient }\end{array}$ & $\begin{array}{l}5.95 \\
\mathrm{mmHg}\end{array}$ \\
\hline & & Septum diastolic & $0.87 \mathrm{~cm}$ & $\begin{array}{l}\text { AO root } \\
\text { dimension }\end{array}$ & $\begin{array}{l}3.7 \\
\mathrm{~cm}\end{array}$ & MV E/A ratio & 0.89 & $\begin{array}{l}\text { Estimated } \\
\text { RVSP }\end{array}$ & $22 \mathrm{mmHg}$ \\
\hline & & $\begin{array}{l}\text { LV volume } \\
\text { diastolic }\end{array}$ & $162 \mathrm{~mL}$ & LA/aorta & 0.86 & $\begin{array}{l}\text { MV peak } \\
\text { gradient }\end{array}$ & $\begin{array}{l}1.06 \\
\mathrm{mmHg}\end{array}$ & $\begin{array}{l}\text { PV peak } \\
\text { velocity }\end{array}$ & $1.14 \mathrm{~m} / \mathrm{s}$ \\
\hline & & LV FS & $26.42 \%$ & LA dimension & $\begin{array}{l}3.2 \\
\mathrm{~cm}\end{array}$ & $\begin{array}{l}\text { MV } \\
\text { deceleration } \\
\text { time }\end{array}$ & $197 \mathrm{~ms}$ & $\begin{array}{l}\mathrm{PV} \text { peak } \\
\text { gradient }\end{array}$ & $\begin{array}{l}5.2 \\
\mathrm{mmHg}\end{array}$ \\
\hline & & $\begin{array}{l}\text { LV EDV/LV EDV } \\
\text { index }\end{array}$ & $\begin{array}{l}162 \mathrm{~mL} / 69 \\
\mathrm{~m}^{2}\end{array}$ & & & MV E' velocity & $0.06 \mathrm{~m} / \mathrm{s}$ & LVOT VTI & $13.5 \mathrm{~cm}$ \\
\hline & & $\begin{array}{l}\text { LV ESV/LV ESV } \\
\text { index }\end{array}$ & $\begin{array}{l}64.5 \\
\mathrm{~mL} / 27 \mathrm{~m}^{2}\end{array}$ & & & Estimated RAP & $\begin{array}{l}10 \\
\mathrm{mmHg}\end{array}$ & $\begin{array}{l}\text { Estimated } \\
\text { PASP }\end{array}$ & $\begin{array}{l}21.56 \\
\mathrm{mmHg}\end{array}$ \\
\hline & & & & & & TR velocity & $1.70 \mathrm{~m} / \mathrm{s}$ & & \\
\hline & & & & & & TR gradient & $\begin{array}{l}11.56 \\
\mathrm{mmHg}\end{array}$ & & \\
\hline
\end{tabular}

\section{TABLE 1: Transthoracic echocardiography results}

The findings showed mild mitral annular calcification, trivial mitral regurgitation, normal left ventricle structure and function.

TTE: transthoracic echocardiogram; EF: ejection fraction; LV: left ventricle; PW: posterior wall; FS: fractional shortening; EDV: end diastolic volume; ESV: end systolic volume; AO: aorta; LA: left atrium; MV: mitral valve; RAP: right atrial pressure; TR: tricuspid regurgitation; AV: aortic valve; RVSP: right ventricular systolic pressure; PV: pulmonic valve; LVOT: left ventricular outflow tract; VTI: velocity time integral; PASP: pulmonary artery systolic pressure

Initial laboratory studies can be found in Table 2 . 


\section{Cureus}

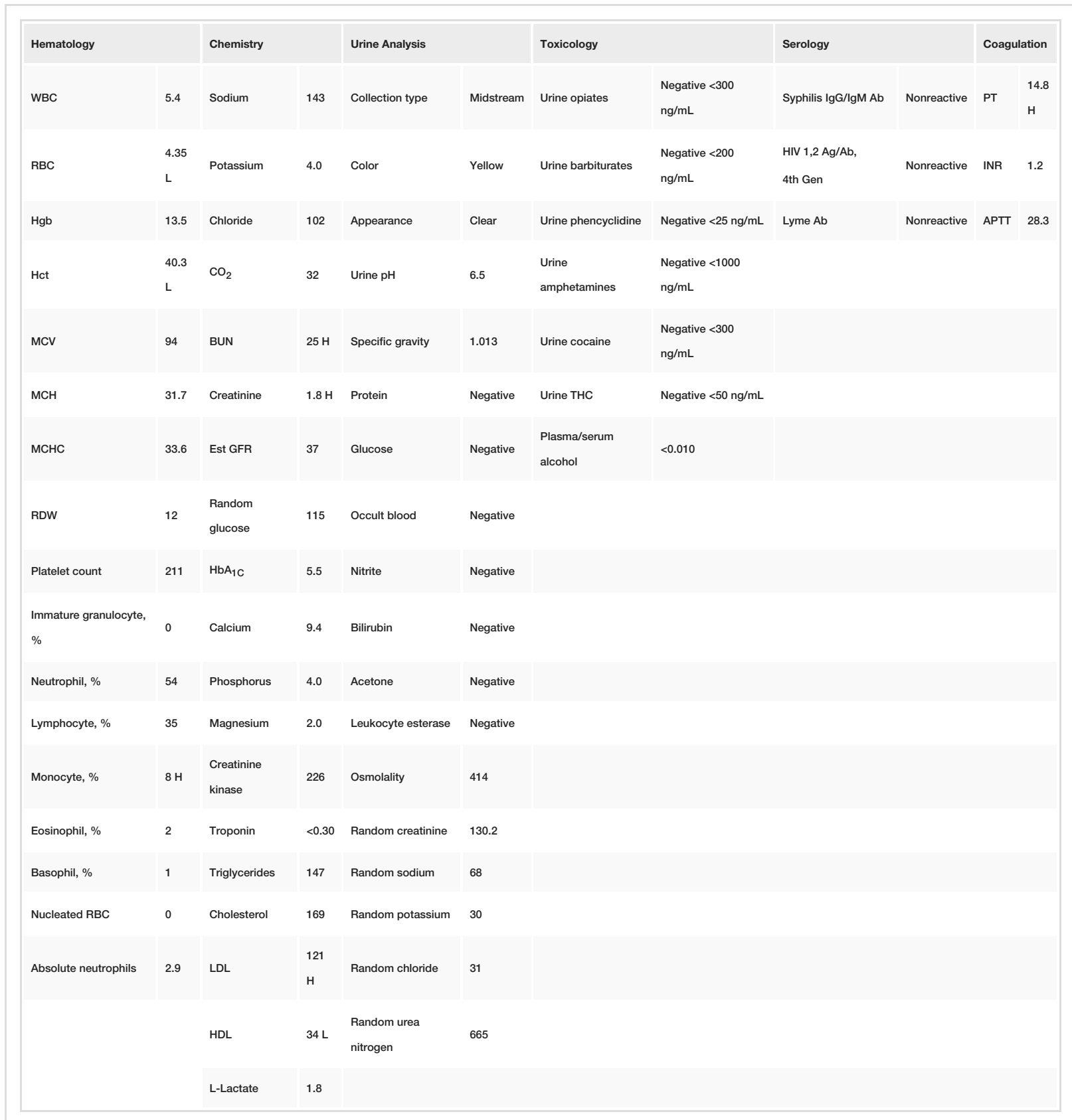

\section{TABLE 2: Laboratory results}

H: high value (above normal limits); L: low value (below normal limits); WBC: white blood cell count; RBC: red blood cell count; Hgb: hemoglobin; Hct: hematocrit; MCV: mean corpuscular volume; MCH: mean corpuscular hemoglobin; MCHC: mean corpuscular hemoglobin concentration; RDW: red cell distribution width; PT: prothrombin time; INR: international normalized ratio; APTT: activated partial thromboplastin time; $\mathrm{CO}_{2}$ : carbon dioxide; Est: estimated; $\mathrm{HbA}_{1 \mathrm{c}}$ : hemoglobin $\mathrm{A}_{1 \mathrm{c}}$; LDL: low-density lipoprotein; HDL: high-density lipoprotein; THC: tetrahydrocannabinol; Ig: immunoglobulin; Ab: antibody; Ag: antigen; Gen: generation

The patient was found to have a kidney injury which was probably chronic and secondary to his hypertension. Elevated prothrombin time was attributable to warfarin; however, the international normalized ratio was subtherapeutic. First-degree atrioventricular block on ECG was attributed to betablocker compliance and the pedal edema to amlodipine, a dihydropyridine calcium-channel blocker. Neurology recommended a five-day trial of prednisone in case the symptoms were secondary to an autoimmune or inflammatory etiology; however, the patient exhibited no response and MRI results suggested an alternative diagnosis.

The patient was discharged following a negative stroke and traumatic head injury work-up with an outpatient audiogram appointment. At time of discharge and otolaryngology follow-up visit, the diagnosis was consistent with superficial siderosis. The patient did not meet Parkinson's criteria, and he was 


\section{Discussion}

Superficial siderosis is a rare and frequently neglected cause of sensorineural hearing loss and progressive ataxia in the elderly $[3,4,9]$. It develops secondary to slow and repeated intracranial hemorrhages into the subarachnoid space, which result in chronic intra- and extracellular hemosiderin deposition in the subpial layers of the brain, spinal cord, and CNs $[3,6,7,9]$. Possible causes of these bleeds include intracranial tumors, head trauma, arteriovenous malformations, aneurysms, cervical root avulsion, neurosurgical procedures, brachial plexus injury, amyloid angiopathy, and chronic subdural hematomas [6-8]. Hemosiderin is most commonly found surrounding the brain stem, cerebellum, and basal cisterns as it pools in the posterior fossa, although superficial cortical deposition can be seen as well $[1,5,9]$. The diagnostic procedure of choice is T2 and susceptibility-weighted (SW) MRI, which visualizes paramagnetic blood products as hypointense [5,9]. T1 and gradient echo MRI are less sensitive in detecting blood products [5]. CT is not ideal for detecting hemosiderin, but these scans can be used to detect hemosiderin as hyperintense or to rule out other substances such as calcium that can appear as hypointense on T2 and SW MRI [9].

Superficial siderosis preferentially disturbs tissues with greater exposure to CSF and longer glial segments. This increases the rate of iron overload and subsequent lipid peroxidation of surrounding structures [3]. The condition has an atypical triad of impairment, which includes bilateral sensorineural hearing loss owing to the nature and long time course of CN VIII injury around the basal cisterns (Figure 2); gait ataxia due to involvement of the cerebellar folia and vermis (Figure 2); and myelopathy due to pyramidal tract involvement $[3,8,9]$. Other symptoms can include anosmia, which is often overlooked owing to the long CN I glial sheath; dementia due to cortical necrosis; cerebral atrophy; and declining executive function $[3,9]$.

Hearing loss in this age group is often attributed to presbycusis, and further investigation is curtailed [4]. However, the presence of additional symptoms should raise a strong clinical suspicion for an alternative ongoing disease process. Differential diagnoses should include multiple sclerosis, autoimmune disease, neurosyphilis, Lyme disease, ototoxic pharmaceuticals (salicylates, aminoglycosides, platinum-based chemotherapeutic agents, etc.), and superficial siderosis [4,7]. It is less likely for Parkinson's disease to be confused with siderosis. We recommend increasing suspicion for individuals on anticoagulation and individuals with prior head injuries.

Bleeding sources are identified in less than $50 \%$ of cases $[4,9]$. Management is approached stepwise, with a primary goal of stopping the bleeding, and secondary goals focused on chelation of hemosiderin deposits with lipid-soluble agents such as deferoxamine, deferiprone, and trientine. However, the risks often outweigh the benefits as symptomatic improvement can be negligible $[7,8]$.

\section{Conclusions}

Superficial siderosis should be considered in any case of progressive bilateral sensorineural hearing loss and ataxia with or without additional accompanying symptoms, especially in individuals on long-term anticoagulation or those with prior head injuries. It is commonly misdiagnosed or underdiagnosed owing to its low frequency of occurrence in the general population. Treatment entails surgical correction of the hemorrhage, followed by iron chelation should benefits outweigh risks. We recommend increasing clinical sensitivity in order to decrease time to diagnosis and improve overall patient outcomes.

\section{Additional Information \\ Disclosures}

Human subjects: Consent was obtained by all participants in this study. Conflicts of interest: In compliance with the ICMJE uniform disclosure form, all authors declare the following: Payment/services info: All authors have declared that no financial support was received from any organization for the submitted work. Financial relationships: All authors have declared that they have no financial relationships at present or within the previous three years with any organizations that might have an interest in the submitted work. Other relationships: All authors have declared that there are no other relationships or activities that could appear to have influenced the submitted work.

\section{References}

1. Pichler M, Vemuri P, Rabinstein AA, et al.: Prevalence and natural history of superficial siderosis a population-based study. Stroke. 2017, 48:3210-3214. 10.1161/STROKEAHA.117.018974

2. Vernooij MW, Ikram MA, Hofman A, Krestin GP, Breteler MMB, Van Der Lugt A: Superficial siderosis in the general population. Neurology. 2009, 73:202-205. 10.1212/WNL.0b013e3181ae7c5e

3. Kale SU, Donaldson I, West RJ, Shehu A: Superficial siderosis of the meninges and its otolaryngologic connection: a series of five patients. Otol Neurotol. 2003, 24:90-95. 10.1097/00129492-200301000-00019

4. Parnes SM, Weaver SA: Superficial siderosis of the central nervous system: a neglected cause of sensorineural hearing loss. Otolaryngol Neck Surg. 1992, 107:69-77. 10.1177/019459989210700111

5. Robinson RJ, Bhuta S: Susceptibility-weighted imaging of the brain: current utility and potential 


\section{Cureus}

applications. J Neuroimaging. 2011, 21:189-204. 10.1111/j.1552-6569.2010.00516.x

6. Kondziella D, Lindelof M, Haziri D, Larsen VA, Kruse A: Diagnostic and therapeutic challenges in superficial CNS siderosis. Dan Med J. 2015, 62:3-6.

7. Vibert D, Häusler R, Lövblad KO, Schroth G: Hearing loss and vertigo in superficial siderosis of the central nervous system. Am J Otolaryngol. 2004, 25:142-149. 10.1016/j.amjoto.2003.10.001

8. Levy M, Turtzo C, Llinas RH: Superficial siderosis: a case report and review of the literature . Nat Clin Pract Neurol. 2007, 3:54-58. 10.1038/ncpneuro0356

9. Kumar N, Cohen-Gadol A, Wright R, Miller G, Piepgras D, Ahlskog J: Superficial siderosis. Neurology. 2006, 66:1144-1152.

10. Pichler M, Vemuri P, Rabinstein AA, et al.: Prevalence and natural history of superficial siderosis a population-based study. Stroke. 2017, 48:3210-3214. 10.1161/STROKEAHA.117.018974 